Macedonian Pharmaceutical Bulletin, 66 (Suppl 1) 165 - 166 (2020)

Online ISSN 1857 - 8969

UDC: $615.11 .012: 614.35$

DOI: 10.33320/maced.pharm.bull.2020.66.03.082

Short communication

\title{
Some challenges in global clinical development of generic products
}

\author{
Rozeta Mileva Peceva $^{1 *}$, Jasminka Patcheva ${ }^{2}$, Snezana Petrovska $^{1}$ \\ ${ }^{1}$ Alkaloid AD Skopje, Blvd. Aleksandar Makedonski 12, 1000, Skopje, R.N. Macedonia \\ ${ }^{2}$ Pharmaceutical Chamber of Macedonia, 50-ta Divizija, 1000, Skopje, R.N. Macedonia
}

\section{Introduction}

The regulatory agencies globally define the regulatory requirements for the approval of simple small-molecule generic products (Hornecker et al., 2009). Following the globalization of the pharmaceutical supply chain, from clinical trials to drug development and manufacturing, the harmonization of pharmaceutical regulations is essential. Significant progress on industry globalization without regulatory harmonization impacts manufacturers and patients (Alvaro et al., 2019). Many regulatory agencies have limited resources, are lacking sufficient skilled staff and are challenged to stay abreast of rapid advances in new treatments and new technologies (Elias and Hamburg, 2016). Meanwhile companies are often required to conduct similar but distinct studies and submit multiple application for given product to agencies in different countries, increasing the time and the cost it takes to bring new drug to market (Weisfeld and Lustig, 2013). For example, in a case of a generic application when supportive bioequivalence studies are needed, FDA lists as RLD (reference listed drugs) the product which is to be used as a comparator in the bioequivalence study. However, even if the same medicinal product is to be used in a bioequivalence study for EU submission, it must be purchased from the EU market. On the other hand, Canada accepts a drug product purchased in another country which complies with the certain criteria to be considered acceptable to the Minister for use as a Canadian Reference Product (HC, 2017). A solution in such scenario is that generic companies conduct separate bioequivalence studies for each region.

\section{Aims and objectives}

This paper has intention to point out some similarities and differences in the recommendation of EMA, FDA and Health Canada (HC) on some aspects on bioequivalence studies, which affect the generic industry during clinical drug development process of oral immediate release formulations.

\section{Materials and methods}

Analysis of several aspects on the requirements for the bioequivalence studies for oral immediate release formulations of relevant guidelines from of EMA, FDA and $\mathrm{HC}$ has been conducted. The following Guidelines were analyzed:

1. FDA, Guidance for Industry Bioavailability and Bioequivalence Studies for Orally Administered Drug Products - General Considerations. 2003;

2. FDA, Bioavailability and Bioequivalence Studies Submitted in NDAs or INDs - General Considerations. Draft Guidance. 2014;

3. EMA, Committee for Medicinal Products for Human Use. Guideline on the Investigation of

\footnotetext{
* rozeta.mileva@gmail.com
} 
Bioequivalence. CPMP/EWP/QWP/1401/98 rev. $1 /$ corr $* * 2010$.

4. HC, Guidance document. Conduct and analysis of comparative bioavailability studies. 2012.

\section{Results and discussion}

The results of the analysis have shown that although the analyzed Guidelines from the 3 regions (EU, USA and Canada) are consistent regarding the recommendation to conduct the bioequivalence studies in healthy subjects above 18 years old (in case there are no safely issues), there are differences regarding several aspects, including subject's gender and race. Unlike FDA Guidelines, where the study population should be representative of the general population in terms of age, sex and race the EMA and Health Canada Guideline do not specify the gender and race of the study subjects. Furthermore, according to FDA recommendation, the generic drug product should be generally tested under both fasting and fed conditions. On the other hand, in order to fill in for an application to EU or Canada, the dossier of an immediate release product may be supported generally with one bioequivalence study under fasting conditions, in case the drug is taken under an empty stomach or irrelevant to food intake. As for the sample size, the EMA and $\mathrm{HC}$ guideline sets the minimum required subjects at 12 completers, whereas FDA instructs for adequate statistical power for a BE demonstration. According to EMA guidance, the over-night fasting time is at least $8 \mathrm{~h}$, the same as $\mathrm{HC}$, and the volume of fluid to be taken with the treatments is identified as at least of $150 \mathrm{~mL}$ (EMA, 2010). FDA recommends at least 10 hours overnight fasting before drug administration with $240 \mathrm{~mL}$ of water, and as per $\mathrm{HC}$, the medication is to be taken with 150 to $250 \mathrm{~mL}$ of water and at a standard temperature.

\section{Conclusion}

The present article has highlighted some similarities and differences in the requirements of the guidelines on bioequivalence between FDA, EMA and HC. There are some similarities, but there are also differences in several aspects. Facing the fact that it is difficult to predict global regulatory landscape in the near future, the need of pragmatic and step - wise approach in harmonization is more than necessary. The generic industry should be aware for the above at early stage of drug product development, in order to create appropriate clinical strategy and save resources.

\section{References}

Alvaro, D., Challener. C.A., Branch, E., 2019. From Regulatory Convergence to Global Regulatory Harmonization. Pharma's Almaanac. PAP-Q3-19-NI004.

Elias, Z., Hamburg, M., 2016, The need for global regulatory harmonization: A public health imperative. Science Translational Medicine 8, 338.

EMA, Committee for Medicinal Products for Human Use. Guideline on the Investigation of Bioequivalence. CPMP/EWP/QWP/1401/98 rev. 1/ corr ** 2010. 127.

FDA, Guidance for Industry Bioavailability and Bioequivalence Studies for Orally Administered Drug Products - General Considerations. 2003, 1-26.

FDA, Bioavailability and Bioequivalence Studies Submitted in NDAs or INDs - General Considerations. Draft Guidance, 2014, 1-29.

HC, Guidance document. Use of a Foreign-sourced Reference Product as a Canadian Reference Product, 2017, 1-13.

HC, Guidance document. Conduct and analysis of comparative bioavailability studies, 2012, 1-46.

Hornecer, J.R., 2012. Generic drugs: history, approval process, and current challenges. US Pharm. 34(6), 26-30.

Weisfeld, V., Lustig, T.A., 2013. International Regulatory Harmonization Amid Globalization of Drug Development: Workshop Summery. Forum on drug Discovery, Development and Translation. National Academic Press (US), Washington (DS). 